\section{MS5-P61 Molecular analysis of a G-actin} sensor

Stephane Mouilleron ${ }^{1}$

1. Francis Crick Institute, London, UK

email: stephane.mouilleron@ crick.ac.uk

Actin dynamics control many aspects of cell shape and cell motility through regulatory interactions with a large variety of actin-binding proteins. Signalling to these actin regulators frequently involves a Rho GTPase-stimulated pathway that leads to a dramatic fluctuation in the levels of monomeric actin (G-actin) following polymerisation to F-actin. Recent studies have identified a molecular G-actin sensor called the RPEL domain that links RPEL-containing proteins and their subcellular localisation to actin dynamics. The RPEL domain contains a tandem array of typically three RPEL motifs, each of which is competent to bind a G-actin molecule (1). The domain is present in two otherwise unrelated protein families; the MRTF family of serum response factor (SRF) transcriptional co-activator proteins and the Phactr family of actin and PP1 phosphatase-binding proteins. We have begun to investigate how the RPEL domain operates in both of these protein contexts and how it modulates subcellular localisation, transcriptional regulation and actomyosin contractility. To define the molecular basis for the sensor we have reconstituted pentameric and trimeric G-actin complexes with the RPEL domain from both MRTF-A and Phactr and used crystallography to reveal discrete supramolecular assemblies with repetitive arrangements of the G-actin subunits around the "crankshaft"-shaped RPEL domain (2). These arrangements are quite different from F-actin intermolecular contacts and are quite unexpected. Our crystal structures reveal cooperative loading of G-actin onto the RPEL domain that we show by several cell-based reporter assays to be of functional importance. These structures explain how G-actin interaction alters the subcellular localisation of both MRTF-A and Phactr by inhibiting nuclear import through competing with importin $\mathrm{a}-\mathrm{b}$ binding (2).

Keywords: actin, RPEL, RhoGAP, NLS, Phactr,
MS5-P62 Structural insights into the pathology of Erwinia amylovora, the causative agent of Fire Blight

Joseph D. Bartho ${ }^{1,2}$, Marco Salomone-Stagni ${ }^{1}$, Dominico Bellini ${ }^{2}$, Jochen Wuerges ${ }^{1}$, Mirco Toccafondi ${ }^{1}$, Armin Schmitt ${ }^{1}$, Nicola Demitri ${ }^{3}$, Martin A. Walsh ${ }^{2}$, Stefano Benini ${ }^{1}$

1. Faculty of Science and Technology, Free University of Bolzano, Piazza Università 5, Bolzano, Italy

2. Diamond Light Source, Harwell Science and Innovation Campus, Didcot, Oxfordshire, OX110DE, United Kingdom

3. ELETTRA Sincrotrone Trieste S.C.p.A., Basovizza, Italy

email: josephdale.bartho@natec.unibz.it

Erwinia amylovora is a gram negative bacterial pathogen of Rosaceae plants, known to infect over 100 species, but most devastating to cultivated apple and pear. Current control measures can reduce the rate of new infections, but the only effective control of existing infections is destruction of the infected plants, resulting in significant economic losses to pome fruit industries. As antibiotic spraying is banned in Europe, and antibiotic resistance is becoming more prevalent in other areas, there is increasing demand for new control measures.

Studying the molecular basis of infection is essential for identifying and understands which genes are important for infection and their mechanisms of overcoming host resistance, and can provide a platform for the development of new targeted control measures to effectively manage this disease.

Infection associated proteins have been investigated based on their importance to the multiple molecular pathways required for successful infection. These include proteins involved in protein secretion, sugar metabolism, siderophore synthesis, exopolysaccharide synthesis, and pathogenic effector proteins. The structural analysis of these proteins by X-ray crystallography have revealed new insights into their roles in infection and plant resistance, along with further potential applications in industry. A selection of these results relating to the various infection pathways will be presented.

Keywords: Protein structure, effector protein, pathogenicity, Erwinia 